This item is the archived peer-reviewed author-version of:

\title{
Management and orchestration challenges in network functions virtualization
}

\section{Reference:}

Mijumbi Rashid, Serrat Joan, Gorricho Juan-Luis, Latré Steven, Charalambides Marinos, Lopez Diego.- Management and orchestration challenges in network functions virtualization

IEEE communications magazine / Institute of Electrical and Electronics Engineers [New York, N.Y.] - ISSN 0163-6804 54:1(2016), p. 98-105

Full text (Publisher's DOI): http://dx.doi.org/doi:10.1109/MCOM.2016.7378433

To cite this reference: http://hdl.handle.net/10067/1444810151162165141 


\title{
Management and Orchestration Challenges in Network Function Virtualization
}

\author{
Rashid Mijumbi, Joan Serrat, Juan-Luis Gorricho, Steven Latré, Marinos Charalambides and Diego Lopez
}

\begin{abstract}
Network Function Virtualization (NFV) continues to draw immense attention from researchers in both industry and academia. By decoupling Network Functions (NFs) from the physical equipment on which they run, NFV promises to reduce Capital Expenses (CAPEX) and Operating Expenses (OPEX), make networks more scalable and flexible, and lead to increased service agility. However, despite the unprecedented interest it has gained, there are still obstacles that must be overcome before NFV can advance to reality in industrial deployments, let alone delivering on the anticipated gains. While doing so, important challenges associated with network and function Management and Orchestration (MANO) need to be addressed. In this article, we introduce NFV and give an overview of the MANO framework that has been proposed by the European Telecommunications Standards Institute (ETSI). We then present representative projects and vendor products that focus on MANO, and discuss their features and relationship with the framework. Finally, we identify open MANO challenges as well as opportunities for future research.
\end{abstract}

Index Terms-Network function virtualization, cloud computing, software defined networking, management, orchestration.

\section{INTRODUCTION}

In recent years, Telecommunication Service Providers (TSPs) have experienced unceasing dwindling in revenue. This has been attributed, in part, to two main factors. On one hand, the seemingly insatiable traffic demands of subscribers require physical network expansions which are achieved at increased CAPEX and OPEX [1]. On the other hand, competition both among themselves and from Over-The-Top service providers means that TSPs cannot respond to the increased costs with increased subscriber fees.

NFV [2] has been identified as a potential solution to these problems. The main concept of NFV is the decoupling of functionality (NFs) from capacity (the physical infrastructure on which they run). Breaking the bond between NFs and hardware promises several advantages. First, there is a potential for significant reductions in OPEX through more efficient operations, since most maintenance and updates to NFs can be performed remotely and at scale. In addition, the increased flexibility can lead to more efficient utilization of resources

R. Mijumbi is with the Telecommunications Software and Systems Group at Waterford Institute of Technology. Part of this work was done while he was still with the Universitat Politècnica de Catalunya, 08034 Barcelona, Spain

J. Serrat and J.L. Gorricho are with the Network Engineering Department, Universitat Politècnica de Catalunya, 08034 Barcelona, Spain

S. Latré is with the Department of Mathematics and Computer Science, University of Antwerp and iMinds, 2020 Antwerp, Belgium

M. Charalambides is with the Department of Electronic and Electrical Engineering, University College London, UK

D. Lopez is in the Global Chief Technical Office (GCTO) Unit, Telefonica I+D, 28050 Madrid, Spain and hence reductions in CAPEX, since TSPs could use the existing network capacity for more user traffic. Finally, NFV may lead to better service agility by allowing TSPs to deploy and/or support new network services faster and cheaper.

These expectations have made NFV a burgeoning research field. The most notable NFV activities are being led by the ETSI. The main objective of the ETSI NFV group $^{1}$ is to develop standards for NFV as well as share experiences of its development and early implementation. To this end, they have defined the NFV problem, some use cases, a reference architecture and a MANO framework, among others [3].

However, while a lot of progress has been made, there are still many technical challenges, which must be overcome before the gains anticipated from NFV can come to fruition. Among them, MANO challenges have drawn special attention. The reason behind this interest is that MANO is a critical aspect towards ensuring the correct operation of the NFV Infrastructure (NFVI) as well as Virtual Network Functions (VNFs). MANO provides the functionality required for the provisioning of VNFs, and the related operations, such as the configuration of the VNFs and the infrastructure these functions run on. It includes the orchestration and lifecycle management of physical and/or virtual resources that support the VNFs [4]. Just like the decoupled NFs, NFV demands a shift from management models that are device-driven to those that are aware of the orchestration needs of NFs running in a virtualized environment. We believe that for NFV to succeed, the main MANO challenges should be addressed at the current specification phase, rather than later when real large scale deployments commence.

In this article, we survey current efforts that address NFV MANO. In particular, in Section II, we begin by summarizing the MANO framework that has been proposed by the ETSI. We then overview representative projects and vendor products that focus on NFV MANO in Section III and IV, respectively. We classify these projects and products in two ways. First, we map their functionality to the functional blocks of the ETSI MANO framework and then we study their features based on four criteria: (1) Management approach (centralized, distributed, policy-based, self-managed), (2) management functions supported (FCAPS), (3) scope (functions, services, network), and (4) the integration of management with that of Software Defined Networking (SDN) and cloud computing both of which are complimentary and/or enablers of NFV. Finally, we identify open challenges and discuss opportunities for future research in Section V, before concluding the article.

\footnotetext{
${ }^{1}$ http://www.etsi.org/technologies-clusters/technologies/nfv
} 


\section{ETSI MANO FRAMEWORK}

The ETSI MANO framework [4] is shown in Fig. 1. The functional blocks in the framework can be grouped into three main entities: (1) NFV architectural layers, (2) NFV management and orchestration, and (3) network management systems. These entities, as well their constituent functional blocks are connected together using a set of defined reference points $^{2}$. The NFV architectural layers include the NFVI and VNFs. NFVI is the combination of both hardware and software resources, which make up the environment in which VNFs are deployed, while VNFs are implementations of NFs that are deployed on those virtual resources.

\section{A. NFV Management and Orchestration}

The NFV MANO consists of three functional blocks (VIM, NFVO and VNFM) and four data repositories (NS Catalogue, VNF Catalogue, NFV Instances and NFVI Resources).

1) Virtualized Infrastructure Manager (VIM): A VIM manages and controls NFVI physical and virtual resources in a single domain. This implies that an NFV architecture may contain more than one VIM, with each of them managing or controlling NFVI resources from a given infrastructure provider. In principle, a VIM may be specialized in handling a certain type of NFVI resource (e.g. compute-only or storageonly), or could manage multiple types of NFVI resources (e.g. nodes in the NFVI).

2) VNF Manager (VNFM): Each VNF instance is assumed to have an associated VNFM. The VNFM is responsible for the management of the lifecycle of VNFs. A VNFM may be assigned the management of a single or multiple VNF instances of the same or different types, including the possibility of a single VNFM for all active VNF instances for a certain domain.

3) NFV Orchestrator (NFVO): The NFVO is aimed at combining more than one function so as to create end-to-end services. To this end, the NFVO functionality can be divided into two broad categories: (1) resource orchestration, and (2) service orchestration. The first is used to provide services that support accessing NFVI resources in an abstracted manner independently of any VIMs, as well as governance of VNF instances sharing resources of the NFVI infrastructure. Service orchestration deals with the creation of end-to-end services by composing different VNFs, and the topology management of the network services instances.

4) Data Repositories: Data repositories are databases that keep different types of information in the NFV MANO. Four types of repositories can be considered: (1) The NS catalog is a set of pre-defined templates, which define how services may be created and deployed, as well as the functions needed for the service and their connectivity; (2) the VNF catalog is a set of templates which describe the deployment and operational characteristics of available VNFs; (3) the NFVI resources repository holds information about available/allocated NFVI

\footnotetext{
${ }^{2} \mathrm{~A}$ reference point is a conceptual point at the conjunction of two communicating functional entities. A detailed description of all the reference points in the ETSI NFV MANO framework can be found in [4].
}

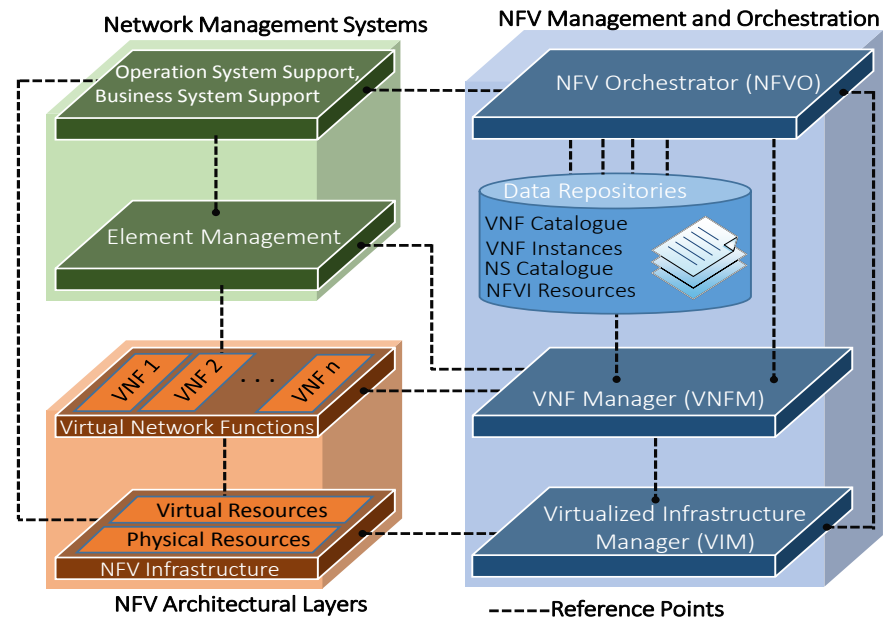

Fig. 1. ETSI NFV MANO Framework

resources; and (4) the NFV instances repository holds information about all function and service instances throughout their lifetime.

\section{B. Network Management Systems}

NFV is not intended to require a drastic change on the current mechanisms of network service provisioning, and aims at a gradual transition from network infrastructures based on physical nodes, easing the integration in a heterogeneous environment. Therefore, network management systems will keep having a key role in NFV, coordinated with the MANO entities by means of a clear separation of roles. MANO entities will deal with those aspects related to the virtualization mechanisms, while network management functions are expected to take care of the features associated to the semantics of the specific network services being provided by the composition of VNFs and, potentially, physical nodes. These network management systems include Element Management (EM), the Operation System Support (OSS), and the Business System Support (BSS).

\section{Projects Related to NFV MANO}

\section{A. CloudNFV}

CloudNFV $^{3}$ is an open platform for implementing NFV based on cloud computing and SDN. The CloudNFV architecture, illustrated in Fig. 2, is made up of 3 main elements: Active virtualization, NFV orchestrator, and NFV Manager. Active virtualization is a data model (based on TM Forum's SID [5]), which represents all aspects of services, functions and resources. It is made up of an active contract and active resource. Active resource describes the status of all resources in the infrastructure, while active contract includes all service templates which define the characteristics of all the available NFs. The orchestrator has policy rules, which, combined with service orders and the status of available resources, determines

\footnotetext{
${ }^{3}$ www.cloudnfv.com/WhitePaper.pdf
} 


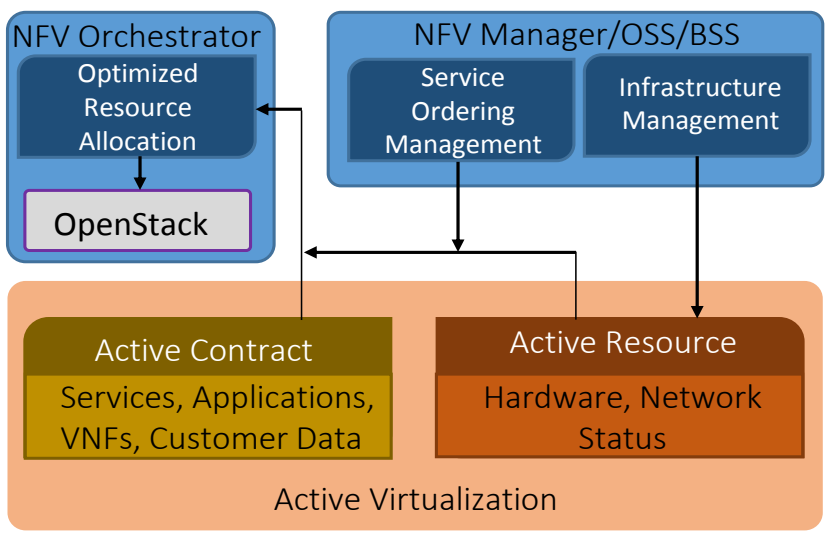

Fig. 2. CloudNFV Architecture

the location of the functions that make up the service as well as connections between them.

After service deployment all resources report their status and traffic to the active resource. The management processes running against active resources allows for reflection of this status using Management Information Bases (MIBs). The main difference between the ETSI NFV MANO and CloudNFV is that unlike the former, the latter considers both management and orchestration as applications that can run off a unified data model.

\section{B. ExperiaSphere}

ExperiaSphere $^{4}$ is a MANO model for NFV, which is based on a combination of open-source tools. ExperiaSphere is founded on the concept of service models. These define how resources expose service features and how the functions decompose to resources. The service models are then used by a broker, who selects one or more required service models to create a service instance. Once the service instance is created, its status is tracked throughout its lifecycle. To achieve these objectives, ExperiaSphere is based on two principles: Structured intelligence and derived operations.

Structured intelligence uses an integration of Universal Service Definition Language (USDL) and Topology and Orchestration Specification for Cloud Applications (TOSCA) [6] to define the relationship between service elements, service goals, and the infrastructure. Derived operations allows for managing virtualized services and resources as if they were physical. The management functions operate on virtual elements of the service, using variables defined per element but derived from the state of real resources.

\section{OpenMANO}

OpenMANO [7] is an open source project led by Telefonica, which is aimed at implementing the ETSI NFV MANO framework, and addressing the aspects related to performance and portability by applying Enhanced Platform Awareness (EPA) ${ }^{5}$ principles. As shown in Fig. 3, the OpenMANO architecture

\footnotetext{
${ }^{4} \mathrm{http}: / / \mathrm{www} . e x p e r i a s p h e r e . c o m /$

${ }^{5}$ https://01.org/sites/default/files/page/openstack-epa_wp_fin.pdf
}

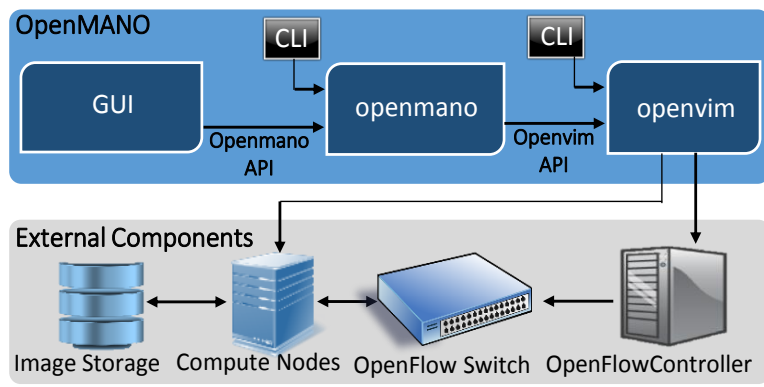

Fig. 3. Telefonica's OpenMANO Architecture

consists of three main components: openmano, openvim, and a graphical user interface (GUI). In addition, there are two command line interfaces (CLI) used to interact with openmano and openvim.

openvim is a lightweight, NFV-specific VIM implementation directly interfacing with the compute and storage nodes in the NFVI, and with an openflow controller in order to create the infrastructural network topology, and enforce the EPA principles mentioned above. It offers a REST-based northbound interface (openvim API) to openmano, where enhanced cloud services are offered including the lifecycle management of images, flavors, instances and networks. The openvim API extends the OpenStack API to accommodate EPA. OpenMANO has a northbound interface (openmano API), based on REST, where MANO services are offered including the creation and deletion of VNF templates, VNF instances, network service templates and network service instances.

\section{OPNFV}

$\mathrm{OPNFV}^{6}$ is an open source project founded and hosted by the Linux Foundation, and composed of TSPs and vendors. The objective is to establish a carrier-grade, integrated, open source reference platform which may be used to validate multi-vendor, inter-operable NFV solutions. OPNFV plans to validate existing standard specifications, contribute improvements to relevant upstream open source projects, and develop necessary new functionality both within OPNFV and upstream projects. In particular, it is focused on implementing the NFV requirements provided by the ETSI. To this end, the first outcome of the project referred to as OPNFV Arno, was released in June 2015. Arno is an initial build of the NFVI and VIM components of the ETSI architecture.

\section{E. ZOOM}

$\mathrm{ZOOM}^{7}$ is a TM Forum project aimed at enabling the deployment of services by automating the provisioning process through improved OSS/BSS models. To achieve this, the project regularly conducts a range of hands-on technology demos each of which is developed from what they call a catalyst project. Each catalyst project is sponsored by one or more network operators and equipment and software vendors

\footnotetext{
${ }^{6} \mathrm{https}: / / \mathrm{www} . o p n f v . o r g /$

${ }^{7}$ https://www.tmforum.org/collaboration/catalyst-program/current-catalysts/
} 
in a real-world demo. The project currently runs about 9 catalysts with a focus on NFV aspects including end-to-end automated management of hybrid networks and demonstrating the impact and value of dynamic security orchestration in an NFV environment.

\section{PRE-STANDARDIZATION NFV MANO PRODUCTS}

\section{A. CloudBand}

Alcatel-Lucent's CloudBand ${ }^{8}$ is an NFV platform, which is comprised of a software and hardware stack with two elements; a node and a management system. The CloudBand node provides the computing, storage, and networking hardware to host cloud services, while the management system is the MANO element of CloudBand. The management system aggregates distributed cloud resources - the nodes - so as to provide a view of the entire NFVI as a single pool. It orchestrates, automates, and optimizes VNFs across the service provider's network and data centers.

\section{B. Ensemble Service Orchestrator}

Overture's Ensemble Service Orchestrator (ESO) ${ }^{9}$ is an NFV service and VNF lifecycle management and orchestration system. The system coordinates and connects virtual resources to physical network elements to create virtualized services across multiple networking layers. ESO supports the placement of VNFs in centralized as well as distributed data centers. It uses the OpenStack cloud controller to manage the virtual compute environment, including virtual machines, virtual switches and data center switches. ESO is the key component of Overture's Ensemble Open Service Architecture (OSA), which is a framework for service MANO.

\section{OpenNFV}

HP's OpenNFV ${ }^{10}$ is an NFV platform that leverages open source technology to provide an open, end-to-end NFV and SDN infrastructure. OpenNFV is aligned towards providing solutions to each of the functional blocks defined in the ETSI NFV reference architecture. With regard to MANO, OpenNFV includes three solutions; NFV Director, NFV Manager, and Helion OpenStack.

The NFV Director is an NFVO that can be used to automate the deployment and monitoring of an VNF ecosystem. It is aimed at ensuring that each VNF can efficiently run on heterogeneous hardware platforms and virtualization environments. VNF managers are responsible for the VNFs lifecycle actions, for example, by deciding to scale up or down. The Helion OpenStack provides an open source cloud platform for running VNFs.

\footnotetext{
${ }^{8}$ http://resources.alcatel-lucent.com/asset/180265

${ }^{9} \mathrm{http}: / / \mathrm{www}$. overturenetworks.com/products/network-virtualization/

${ }^{10} \mathrm{http}: / /$ www $8 . h p . c o m /$ us/en/cloud/nfv-architecture.html
}

\section{Open Network Strategy}

Cisco's Open Network Strategy (OPN) ${ }^{11}$ includes a services orchestrator, a VNF manager, and an SDN controller, all of which are aimed at providing implementations for some of the functional blocks of the ETSI MANO framework. The services orchestrator is responsible for providing the overall lifecycle management at the network service level. It uses model-based work-flows that enable the design of services based on predefined service elements. The VNF Manager provides VNF lifecycle management, including the creation, provisioning, and monitoring of both Cisco and third-party VNFs. Finally, the SDN Controller is responsible for connecting the virtualized services to the service provider VPNs, the Internet, or both.

\section{E. Planet Orchestrate}

Planet Orchestrate is part of Cyan's Blue Planet Suite ${ }^{12}$, which is a SDN and NFV platform aimed at service orchestration, automation, SDN control, and multi-vendor management capabilities. Its functionality is based on the requirements of ETSI's NFV MANO framework. It uses TOSCA templates and information models to define service components and their relationships.

Planet Orchestrate can perform VNF management and orchestration functionality. The NFV orchestration engine performs placement of VNFs and supports distributed NFVI to optimize use of NFV resources. On the management side, it supports the performance, availability and security demands of service provider applications. Performance monitoring and alarm/event reporting is provided for the NFVI and virtual functions. An intrinsic knowledge of the topology and the mapping between application and virtual resources enables fault-isolation and recovery as well as high-availability and resiliency.

Summary: In tables I and II, we summarize current activities towards NFV MANO. In Table I, we map the functionalities of each project or product to the functional blocks of the ETSI NFV reference architecture. We can observe that most projects or products choose to rely on existing infrastructures and cloud systems such as OpenStack for achieving the NFVI and some form of data modeling and storage to model and store VNFs. On the MANO part, it can be noted that almost all propose a solution for each of the three functional blocks VIM, VNFM, and NFVO. The difference however, is in the functionality. This can be observed in Table II, which summarizes the management and orchestration functionality of each project/product based on their description. For this purpose, we define four functionality categories. The management approach classifies them based on whether they are centralized, distributed, policy-based, and automated (selfmanaged). The management function classifies them according to their support for five of the basic management functions -

\footnotetext{
${ }^{11} \mathrm{http} / / / \mathrm{www} . c i s c o . c o m / c / e n /$ us/solutions/collateral/service-

provider/network-functions-virtualization-nfv/white-paper-c11-732123.html

${ }^{12} \mathrm{http} / / /$ www.cyaninc.com/products/blue-planet-sdn-platform
} 
TABLE I

Mapping of State-OF-ThE-ARt Projects And Products to the ETSi MANO

\begin{tabular}{|c|c|c|c|c|c|c|c|c|}
\hline & \multicolumn{2}{|c|}{ NFV Architectural Layers } & \multicolumn{4}{|c|}{ NFV MANO Framework } & \multicolumn{2}{|c|}{ Traditional Management Systems } \\
\hline & NFVI & VNFs & VIM & VNFM & NFVO & Data Repositories & $\begin{array}{l}\text { OSS/BSS } \\
\end{array}$ & EM \\
\hline CloudBand & $\begin{array}{l}\text { Nuage, RedHat, } \\
\text { CloudBand }\end{array}$ & $\begin{array}{l}\text { VNF Modelling } \\
\text { (TOSCA) }\end{array}$ & CloudBand Node & $\begin{array}{l}\text { CloudBand Management } \\
\text { System }\end{array}$ & $\begin{array}{c}\text { CloudBand Management } \\
\text { System }\end{array}$ & $\checkmark$ & & \\
\hline CloudNFV & Active Resource & Active Contract & Infrastructure Manager & OSS/BSS & $\checkmark$ & Active Contract & $\checkmark$ & OSS/BSS \\
\hline ESO & & $\checkmark$ & ENC & ESO & ESO & Database & & \\
\hline ExperiaSphere & Resource Domain & TOSCA, USDL & Infrastructure Manager & $\begin{array}{l}\text { State-action service } \\
\text { lifecycle management }\end{array}$ & $\begin{array}{l}\text { State-action service } \\
\text { lifecycle management }\end{array}$ & $\begin{array}{l}\text { Derived } \\
\text { Operations }\end{array}$ & $\begin{array}{l}\text { State-action service } \\
\text { lifecycle management }\end{array}$ & $\begin{array}{l}\text { Derived } \\
\text { Operations }\end{array}$ \\
\hline OpenMANO & $\checkmark$ & $\checkmark$ & Openvim & & Openmano & & & \\
\hline OPN & & & SDN Overly Controller & $\checkmark$ & Services Orchetsrator & & & \\
\hline OpenNFV & $\checkmark$ & & $\begin{array}{l}\text { HP Helion OpenStack } \\
\text { Carrier Grade }\end{array}$ & $\checkmark$ & HP NFV Director & HP NFV Director & $\checkmark$ & \\
\hline OPNFV & $\checkmark$ & & $\checkmark$ & & & & & \\
\hline Planet Orchestrate & & & & $\checkmark$ & $\checkmark$ & & & \\
\hline ZOOM & & & $\checkmark$ & $\checkmark$ & $\checkmark$ & Shared Catalog & $\begin{array}{l}\text { Order, SLA and billing } \\
\text { management systems }\end{array}$ & \\
\hline
\end{tabular}

FCAPS. We define the management scope to include functions, services and networks. An approach is classified as a network management one if it proposes functionality to manage network nodes and links, while service management applies to an approach that manages both functions and their connectivity or chaining to form a service. Finally, since SDN and cloud computing are very important technologies with regard to NFV, we also categorize a project/product based on its ability to manage the interactions between SDN and/or cloud and NFV.

\section{Challenges AND Research Opportunities}

\section{A. Resource Management}

The servers used to host VNFs have a finite amount of memory, compute and storage capacity. And since in practice these servers may be distributed across multiple domains, interdomain link capacity will also be finite. Therefore, to achieve the economies of scale expected from NFV, physical resources should be efficiently managed. Dynamism, scalability and automation are important features of such resource management. In this context, we identify three main challenges:

1) NFV PoP Locations: The first question is determining the locations of NFV points-of-presence (PoP) [3]. In cases where the VNFs will be hosted in operator network nodes, it is necessary to decide which subset of the nodes can be used as NFV PoPs. As this does not have to be done often, it could be formulated as an optimization problem whose objective considers the (latency to the) location of subscribers, setup and maintenance costs of both servers as well as front haul links in case on virtualized radio access networks.

2) Function Placement: In order to compose a service, its constituent functions must be deployed. Decisions must be made on where functions should be placed among the available PoPs. This problem is related to Virtual Network Embedding (VNE) [8] and hence similar approaches may be applied. To this end, it may be formulated as a mathematical problem with such objectives as load balancing and energy conservation. However, any such formulation should be able to take the function chaining and/or precedence requirements into consideration to avoid network congestion.

In addition, while most current NFV proofs of concept have been based on each VNF being hosted by a dedicated VM, such an approach would not scale especially for light functions such as those which are part of customer premises equipment. In this case, it would be more efficient to host multiple functions in a single VM, by use of containers and dockers. In this case, there would be a need for scheduling approaches, such as [9], for allocating the VM resources.

3) Dynamic Resource Management: One of the selling points of NFV is the ability to scale resources dynamically. There must be capabilities to increase or reduce the amount of resources allocated to specific functions or VMs. While current virtualization or cloud platforms allow for this, many of them require a manual trigger by the user or resource owner. Therefore, automation and self-allocation mechanisms that allow the network to dynamically manage resources are critical to the success of NFV.

\section{B. Distributed Management}

Current MANO approaches mainly focus on centralized solutions, which pose scalability limitations, especially in scenarios where services span across multiple administrative domains. This is mainly attributed to the communication overhead and the delay incurred by the collection and analysis of data associated with a large number of heterogeneous sources, which prevents these processes from being executed frequently. As a result, the lag in learning the state of services and resources does not allow for online reconfiguration operations. To better react to demand dynamics but also to changing service requirements, efficient monitoring mechanisms need to be implemented, which feed distributed management entities with the necessary information to perform dynamic configuration changes. A communication protocol to support lightweight coordination among distributed decision makers, aiming to optimize the usage of resources and the performance of services, is another key research issue. 
TABLE II

Characteristics of State-OF-THE-ARt Projects and Products

\begin{tabular}{|c|c|c|c|c|c|c|c|c|c|c|c|}
\hline & & CloudBand & CloudNFV & ESO & ExperiaSphere & OpenMANO & OPN & OpenNFV & OPNFV & Planet Orchestrate & ZOOM \\
\hline \multirow{4}{*}{$\begin{array}{c}\text { Management } \\
\text { Approach }\end{array}$} & Centralized & $\checkmark$ & $\checkmark$ & $\checkmark$ & $\checkmark$ & $\checkmark$ & $\checkmark$ & $\checkmark$ & $\checkmark$ & $\checkmark$ & $\checkmark$ \\
\hline & Distributed & & & & & & & & & & \\
\hline & Policy-based & $\checkmark$ & $\checkmark$ & $\checkmark$ & $\checkmark$ & $\checkmark$ & $\checkmark$ & $\checkmark$ & & $\checkmark$ & $\checkmark$ \\
\hline & Self-managed & $\checkmark$ & & $\checkmark$ & $\checkmark$ & & $\checkmark$ & $\checkmark$ & & & $\checkmark$ \\
\hline \multirow{5}{*}{$\begin{array}{c}\text { Management } \\
\text { Function } \\
\text { (FCAPS) }\end{array}$} & Fault & & & $\checkmark$ & & & $\checkmark$ & & $\checkmark$ & $\checkmark$ & $\checkmark$ \\
\hline & Configuration & $\checkmark$ & $\checkmark$ & $\checkmark$ & $\checkmark$ & $\checkmark$ & & $\checkmark$ & $\checkmark$ & $\checkmark$ & $\checkmark$ \\
\hline & Accounting & & & & & $\checkmark$ & & & $\checkmark$ & & \\
\hline & Performance & $\checkmark$ & $\checkmark$ & $\checkmark$ & & $\checkmark$ & $\checkmark$ & $\checkmark$ & & $\checkmark$ & $\checkmark$ \\
\hline & Security & $\checkmark$ & & & & & & & & $\checkmark$ & $\checkmark$ \\
\hline \multirow{3}{*}{$\begin{array}{c}\text { Management } \\
\text { Scope }\end{array}$} & Functions & & & $\checkmark$ & & $\checkmark$ & $\checkmark$ & & $\checkmark$ & & $\checkmark$ \\
\hline & Services & $\checkmark$ & $\checkmark$ & $\checkmark$ & $\checkmark$ & $\checkmark$ & $\checkmark$ & & & $\checkmark$ & $\checkmark$ \\
\hline & Network & & & $\checkmark$ & & & & & & $\checkmark$ & $\checkmark$ \\
\hline Managing & SDN & $\checkmark$ & & & & $\checkmark$ & $\checkmark$ & & $\checkmark$ & & \\
\hline Related Areas & Cloud & $\checkmark$ & $\checkmark$ & & $\checkmark$ & & & $\checkmark$ & $\checkmark$ & $\checkmark$ & $\checkmark$ \\
\hline
\end{tabular}

\section{Management of SDN}

While NFV and SDN are not dependent on each other, they are highly related and complimentary. Individually, NFV and SDN introduce high levels of dynamism and variability, which curtails the visibility and control of human operators. Therefore, traditional management approaches must be improved to accommodate each one of them. While some claim to be based on SDN, all the surveyed projects and solutions focus on managing virtualized compute infrastructure/resources and functions. In the same way, from the SDN perspective, the focus is on managing networks in a programmatic way. We are not aware of management solutions that combine both, which is a key research area. In addition, the management of SDN itself still has open questions [10] such as on the number of controllers, their location, and avoiding conflicts in cases where more than one controller manages a given forwarding element. In this case, ideas from policy-based management that have been proposed in most of the surveyed MANO solutions may be extended to SDN.

\section{Security in the Cloud}

As SDN and NFV focus on the remote programmability of network resources and their functions, it opens up an important set of new potential threats and attacks that, if successful, could have a far greater impact than in an nonNFV environment. The ETSI NFV security group has recently drafted a document that defines the possible security threats that NFV brings to the table ${ }^{13}$. The document is a statement regarding possible security problems but does not yet provide a recommendation to tackle them. Partly because of this, security is currently underdeveloped. Of the surveyed projects and solutions, only CloudBand proposes a comprehensive security solution including anomaly prediction, detection and isolation, as well as providing security as a service. In the case of ZOOM and Planet Orchestrate, security support is claimed based on a set of best practices and the integration with another product, respectively.

\footnotetext{
${ }^{13}$ NFV Security; Problem Statement. Bob Briscoe (Rapporteur). Draft Group Specification published, Oct 2014
}

Therefore, real security support is lacking in all NFV products, despite the relevant new threats. Important security challenges in this area are detecting and blocking possible intrusion. Specifically, in multi-vendor environments, there are new security concerns of one TSP competitor having access to another TSP's data/configuration. In such a case, isolation between them is important.

\section{E. Management across the board}

Most solutions provide a way to perform configuration. A limited number add performance management as well and, as discussed above, only a few provide - albeit limited - security management too. In most solutions, accounting management is completely overlooked. As such, there are currently no ways of tracking network utilization to ensure that individual parties can be appropriately billed for their use. This is very contradictory given the openness that NFV promises, especially in introducing a more multi-vendor world where different parties can coexist on the same device through virtualization.

More generally, the management of entire service lifecycle is still missing. One of the unique selling points of NFV is that it promises to automate the entire process of setting up and removing a service (chain), including configuration, performance optimization, response to faults and billing. With the support for accounting missing in all products, this promise has not yet been delivered. One of the reasons is that accounting is often heavily intertwined with legacy solutions. Providing support for all FCAPS functionality is therefore highly challenging but at the same time very important for NFV to really bring a change to the telco world.

\section{F. Programmability and Intelligence}

Given that NFV envisions the deployment and maintenance of complex services across heterogeneous physical resources, a rich set of programmable interfaces should be developed, which will extend the current SDN functionality beyond the scope of controlling simple connectivity resources. Based on the abstraction of the flow, SDN solutions control the distribution of traffic in the network according to forwarding 
rules. Additional abstractions that apply to computation and storage resources are required so that network functions can be instantiated across multiple vendor technologies, but also interfaces that will allow to dynamically (re-)program the configuration of those functions and control, for example, their placement.

A related research challenge concerns the level of intelligence that can be achieved. This is defined by the way in which services are programmed, e.g. declaratively, and the degree by which parameters can be configured. A MANO system should be intelligent enough so that (re-)configuration operations can be automated to a large extend, especially those that react to run-time events. In this respect, intelligent mechanisms that automatically transform high-level policy to operational parameters and perform configuration integrity checks are of paramount importance.

\section{G. Interfacing and Interoperability}

One of the main goals of NFV is to break the bond between equipment vendors and TSPs, and the services they provide. One key requirement for this is support for interoperability. While remarkable progress has been made by the ETSI in defining the NFV MANO framework and the constituent (intra-operator) interfaces, there is still much to be done in terms of defining interfaces aimed at supporting interoperability between different vendors with different functions. Inter-operability problems can already be observed in all the surveyed projects and solutions. For example, while they all are "based on the ETSI MANO framework", each of the projects and solutions surveyed uses a custom model and/or representation for functions and services. This would mean that, unless clear interfaces are defined, it is impossible to chain functions from different operators into a single service. This is because while the ETSI proposes VNF and Network Service descriptors as templates for definition of functions and services, it does not define a data model to realize descriptors.

In this direction, the Alliance for Telecommunications Industry Solutions (ATIS) ${ }^{14}$ has been focused on inter-carrier inter-operability, new service descriptions and automated processes. While ATIS has recently proposed seven inter-provider use cases aimed at the same, these are generally generic descriptions. In particular, they do not define any technical requirements or solutions that could be used to enable the uses cases.

\section{CONCLUSION}

In this article, we presented an overview of the NFV MANO framework that has been recently proposed by ETSI as well as representative projects attempting to realize the framework. Other research projects as well as industry products focused NFV MANO have been surveyed, identifying their functionality as well as their mapping to the ETSI NFV MANO. Based on these, we have identified and discussed open challenges as well as opportunities for research with regard to MANO in NFV.

\footnotetext{
${ }^{14}$ https://www.atis.org/NFV/index.asp
}

We have observed that while ETSI completed the first phase of work, the proposed MANO framework still lacks details and standards on the implementation for both the managers as well as the interfaces. As a result, most of the pre-standardization solutions are in fact customized and based on proprietary solutions, which will likely lead to inter-operability issues. In addition, while some of the identified challenges such as security are being considered in some of the projects and/or industrial products, others such as resource management-and in particular automated resource management-have not received significant attention yet. It is our opinion that the success of NFV will depend, in part, on the availability of mechanisms that are able to autonomously manage network and function resources.

\section{ACKNOWLEDGEMENT}

The authors are indebted to the Editor-in-Chief and the Series Editors for coordinating the review process, and to the anonymous reviewers for their insightful comments and suggestions. This work has been supported in part by FLAMINGO, a Network of Excellence project (318488) supported by the European Commission under its Seventh Framework Programme, the Science Foundation Ireland Research Centre CONNECT (13/RC/2077), and project TEC201238574-C02-02 from Ministerio de Economia y Competitividad.

\section{REFERENCES}

[1] A. Checko, H. Christiansen, Y. Yan, L. Scolari, G. Kardaras, M. Berger, and L. Dittmann, "Cloud ran for mobile networks - a technology overview," Communications Surveys Tutorials, IEEE, vol. 17, no. 1, pp. 405-426, Firstquarter 2015.

[2] R. Mijumbi, J. Serrat, J. Gorricho, N. Bouten, F. De Turck, and R. Boutaba, "Network function virtualization: State-of-the-art and research challenges," Communications Surveys Tutorials, IEEE, vol. PP, no. 99, pp. 1-1, 2015.

[3] ETSI NFV ISG, "ETSI Network Functions Virtualisation (NFV) Industry Standards (ISG) Group Draft Specifications,” http://docbox.etsi.org/ ISG/NFV/Open, December 2014, Accessed on May 26, 2015.

[4] ETSI GS NFV-MAN 001, "Network Functions Virtualisation (NFV); Management and Orchestration," http://www.etsi.org/, December 2014, ETSI Industry Specification Group (ISG).

[5] K. Ogaki, M. Miyazawa, M. Hayashi, and S. Kadono, "Integrating heterogeneous it/network management models using linked data," in Integrated Network Management (IM 2013), 2013 IFIP/IEEE International Symposium on, May 2013, pp. 768-771.

[6] J. Cardoso, T. Binz, U. Breitenbcher, O. Kopp, and F. Leymann, "Cloud Computing Automation: Integrating USDL and TOSCA," in Advanced Information Systems Engineering, ser. Lecture Notes in Computer Science, C. Salinesi, M. Norrie, and s. Pastor, Eds. Springer Berlin Heidelberg, 2013, vol. 7908, pp. 1-16.

[7] D. R. Lopez, "OpenMANO: The Dataplane Ready Open Source NFV MANO Stack," in IETF 92 Meeting Proceedings, Dallas, Texas, USA, March 2015.

[8] N. Chowdhury, M. Rahman, and R. Boutaba, "Virtual network embedding with coordinated node and link mapping," in INFOCOM 2009, IEEE, April 2009, pp. 783-791.

[9] R. Mijumbi, J. Serrat, J.-L. Gorricho, N. Bouten, F. De Turck, and S. Davy, "Design and evaluation of algorithms for mapping and scheduling of virtual network functions," in IEEE Conference on Network Softwarization (NetSoft). University College London, April 2015.

[10] S. Kuklinski and P. Chemouil, "Network management challenges in software-defined networks," IEICE TRANSACTIONS on Communications, Special Section on Management for Flexible ICT Systems and Services, vol. E97-B, no. 99, pp. 2-9, January 2014. 


\section{AUTHORS' BIOGRAPHIES}

Rashid Mijumbi obtained a degree in electrical engineering from Makerere University, Uganda in 2009, and a PhD in telecommunications engineering from the Universitat Politècnica de Catalunya (UPC), Spain in 2014. He is currently a Postdoctoral Researcher in the Telecommunications Software and Systems Group (TSSG) at Waterford Institute of Technology. His research interests are in management of networks and services. Current focus is on management of resources for virtualized networks and functions, cloud computing and software defined networks.

Joan Serrat is a full professor at UPC. He received a degree of telecommunication engineering in 1977 and a $\mathrm{PhD}$ in the same field in 1983 , both from the UPC. He has been involved in several collaborative projects with different European research groups, both through bilateral agreements or through participation in European funded projects. His topics of interest are in the field of autonomic networking and service and network management.

Juan-Luis Gorricho received a telecommunication engineering degree in 1993, and a Ph.D. degree in 1998, both from the UPC. He is currently an associate professor at the UPC. His recent research interests are in applying artificial intelligence to ubiquitous computing and network management; with special interest on using smartphones to achieve the recognition of user activities and locations; and applying linear programming and reinforcement learning to resource management in virtualized networks and functions.

Steven Latré received a MSc. degree and a Ph.D. both in computer science from Ghent University, Belgium. He is currently an assistant professor at the University of Antwerp, Belgium. His research activity focuses on autonomous management and control of both networking and computing applications. He has been involved in several national and European research projects. His recent work has focused on Quality of Experience optimization and management, distributed control and network virtualization.

Marinos Charalambides is a senior researcher at the University College London. He received a BEng in electronic and electrical engineering, an MSc in communications networks and software, and a $\mathrm{PhD}$ in policy-based management, all from the University of Surrey, UK, in 2001, 2002, and 2009, respectively. He has been working in a number of European and UK national projects since 2005, and his research interests include software-defined networking, policy-based management, in-network caching, and online traffic engineering.

Diego Lopez received a MS from the University of Granada in 1985 , and a $\mathrm{PhD}$ degree from the University of Seville in 2001. He joined Telefonica I+D in 2011 as a senior technology expert and is currently in charge of the Technology Exploration activities within the GCTO Unit. $\mathrm{He}$ is focused on network virtualization, infrastructural services, network management, new network architectures, and network security. He actively participates in the ETSI ISG on NFV (chairing its Technical Steering Committee), the ONF, and the IETF WGs connected to these activities. 